\title{
Puerarin prevents tumor necrosis factor- $\alpha$-induced apoptosis of PC12 cells via activation of the PI3K/Akt signaling pathway
}

\author{
FENG LIANG and SHENGGAO XIE \\ Department of Clinical Biochemistry, School of Laboratory Medicine, Hubei University of Chinese Medicine, \\ Wuhan, Hubei 430065, P.R. China
}

Received March 30, 2016; Accepted March 3, 2017

DOI: $10.3892 /$ etm.2017.4545

\begin{abstract}
Tumor necrosis factor- $\alpha$ (TNF- $\alpha)$, a potential proinflammatory cytokine, is an important component involved in neuronal apoptosis associated with neuroinflammation in the central nervous system. It has been reported that puerarin possesses pharmacological effects, such as anti-apoptotic, antioxidant, anti-osteoporosis, anti-inflammatory, cardioprotective and neuroprotective actions. The aim of the present study was to explore the effect of puerarin on apoptosis induced by TNF- $\alpha\left(3 \times 10^{5} \mathrm{U} / \mathrm{l}\right)$ and its detailed mechanisms in PC12 cells. MTT and flow cytometric assays were performed to evaluate cell cytotoxicity and apoptosis, respectively. An enzymatic assay was used to detect the activity of caspase-3 and caspase-9. Western blot analysis was performed to assess changes in the levels of proteins, including B-cell lymphoma 2 (Bcl-2), Bcl-2-associated X protein (Bax), caspase-3, Akt and phosphorylated Akt. The results showed that puerarin (25 and $50 \mu \mathrm{M}$ ) significantly suppressed TNF- $\alpha$-induced apoptosis in PC12 cells. The TNF- $\alpha$-induced in crease in the $\mathrm{Bax} / \mathrm{Bcl}-2$ ratio was markedly inhibited by pre-treatment with puerarin for $2 \mathrm{~h}$. In addition, puerarin decreased the level of TNF- $\alpha$-induced cleaved caspase-3. Furthermore, puerarin inhibited the TNF- $\alpha$-induced decrease in the phosphorylation of Akt, which was abolished by LY294002, a phosphatidylinositol 3-kinase (PI3K) inhibitor, suggesting that the PI3K/Akt pathway participated in the suppressive effect of puerarin. Taken together, these findings indicated that puerarin prevented TNF- $\alpha$-induced apoptosis in PC12 cells via activating of the PI3K/Akt signaling pathway, suggesting that puerarin may be a potential neuroprotective drug in the clinical treatment of neuroinflammation via anti-apoptotic mechanisms.
\end{abstract}

Correspondence to: Professor Shenggao Xie, Department of Clinical Biochemistry, School of Laboratory Medicine, Hubei University of Chinese Medicine, 1 Huangjiahu West Road, Wuhan, Hubei 430065, P.R. China

E-mail: xie-shenggao@outlook.com

Key words: puerarin, PC12 cells, TNF- $\alpha$, apoptosis, PI3K/Akt pathway

\section{Introduction}

Neuroinflammation is defined as activation of the innate immune system of the brain in response to an inflammatory challenge and is characterized by microglial activation, astrogliosis and neuronal loss (1). In the central nervous system (CNS), tumor necrosis factor- $\alpha$ (TNF- $\alpha$ ), a key proinflammatory cytokine, exerts homeostatic as well as pathophysiological effects. Under normal conditions, TNF- $\alpha$ exerts regulatory functions on physiological processes such as synaptic plasticity, synaptic transmission, learning and memory. Under pathological conditions, microglia and a proportion of astrocytes and oligodendrocytes secrete TNF- $\alpha$, which is an important component of neuronal apoptosis associated with neuroinflammation (2). Neuronal apoptosis is the most crucial event in neuronal loss associated with neurological diseases and is regulated by pro- and anti-apoptotic factors (3). At present, it is urgent to seek the underlying molecular mechanisms of neuronal apoptosis induced by neuroinflammation.

PC12 cells, which originate from a rat adrenal medullary tumour (pheochromocytoma), have been widely used as a neuronal model system to assess processes such as neuronal differentiation, neurite outgrowth, neuronal toxicity and neuronal apoptosis (4-6). Puerarin, known as Ge-gen in Chinese, is isolated from Radix puerariae and has protective effects on the nervous and cardiovascular system to prevent osteoporosis, liver injury and inflammation (7). An increasing body of evidence has indicated that puerarin has anti-apoptotic effects and mitigated apoptosis in multiple cell types, including neurons, microglia, osteoblasts and cardiomyocytes (8-11). A previous study reported that puerarin protected PC12 cells against $\beta$-amyloid-induced cell injury, which was associated with its antioxidant effects (12). Another study confirmed that puerarin protected differentiated PC12 cells from $\mathrm{H}_{2} \mathrm{O}_{2}$-induced apoptosis via Akt phosphorylation (13). However, little is known regarding the effect of puerarin on PC12 cell apoptosis induced by TNF- $\alpha$ and its underlying mechanisms.

The present study demonstrated that puerarin prevented TNF- $\alpha$-induced apoptosis in PC12 cells via activation of the phosphoinositide-3 kinase (PI3K)/Akt signaling pathway to provide potential mechanisms underlying the neuroprotective effect of puerarin, which may be used as a novel neuroprotective agent against neuroinflammation. 


\section{Materials and methods}

Materials. Rat PC12 (adrenal gland pheochromocytoma) cells were purchased from the Cell Bank of the Chinese Academy of Sciences (Shanghai, China). Rat TNF- $\alpha$ was obtained from R\&D Systems (Minneapolis, MN, USA). The specific PI3K/Akt inhibitor LY294002 $(10 \mu \mathrm{M})$ was from Sigma-Aldrich (Merck KGaA, Darmstadt, Germany). Puerarin (purity, $>98 \%$ ) was purchased from the National Institute for the Control of Pharmaceutical and Biological Products (Beijing, China) and dissolved in dimethylsulfoxide (DMSO) at $10 \mathrm{mM}$.

PC12 cell culture. PC12 cells were cultured in Dulbecco's modified Eagle's medium (Hyclone; GE Healthcare, Chalfont, UK) supplemented with $10 \%$ fetal bovine serum (Gibco; Thermo Fisher Scientific, Inc., Waltham, MA, USA) and antibiotics (100 units/ml penicillin and 100 units/ml streptomycin; Gibco, Thermo Fisher Scientific, Inc.) at $37^{\circ} \mathrm{C}$ in a humidified incubator with $5 \% \mathrm{CO}_{2}$. Cells were supplied with fresh medium three times per week, and were split at a 1:3 ratio twice per week.

MTT assay. PC12 cells were seeded into 96-well plates at $1 \times 10^{4}$ cells/well. Puerarin was added at the desired concentration for $2 \mathrm{~h}$ prior to treatment with TNF- $\alpha$, after which the cells were incubated for an additional $24 \mathrm{~h}$. MTT (Sigma-Aldrich) working solution $(0.5 \mathrm{mg} / \mathrm{ml})$ was added to each well, followed by incubation for $4 \mathrm{~h}$ at $37^{\circ} \mathrm{C}$. Subsequently, DMSO (Sigma-Aldrich) was added. The optical density (OD) was measured at a wavelength of $570 \mathrm{~nm}$ using a plate reader (Bio-Rad Laboratories, Inc., Hercules, CA, USA). The inhibitory rate of PC12 cell proliferation was calculated as follows: Inhibitory rate $(\%)=\left(\mathrm{OD}_{\text {control group }}-\mathrm{OD}_{\text {test group }}\right) \times 100 \% / \mathrm{OD}_{\text {control group }}$.

Flow cytometric analysis. Flow cytometric analysis was used to detect the apoptotic rate of cells processed with a fluorescein isothiocyanate (FITC) Apoptosis Detection kit (BD Pharmingen, San Jose, CA, USA). PC12 cells $\left(5 \times 10^{5}\right)$ were collected from 24-well culture plates at the end of the drug incubation. Cells were then washed and incubated in buffer containing $5 \mu 1$ FITC-Annexin $\mathrm{V}$ and $5 \mu 1$ propidium iodide (PI). The mixture was then incubated for $5 \mathrm{~min}$ at room temperature in the dark and immediately analyzed with a flow cytometer (BD FACSVerse) followed by analysis with BD FASCuite software (BD Biosciences, Franklin Lakes, NJ, USA).

Enzymatic assay for caspase-3 and caspase-9 activity. Caspase activity was detected using the caspase- 3 and caspase-9 Activity Assay kit (KeyGen Biotech Co., Ltd., Nanjing, China) according to standard procedures. Cells were collected and extracted on ice in lysis buffer. The protein content of the supernatant was subsequently measured using the bicinchoninic acid (BCA) method with an assay kit (Thermo Fisher Scientific, Inc.). An equal amount of protein $(50 \mu \mathrm{g})$ was detected using reaction buffer containing chromogenic substrates at $37^{\circ} \mathrm{C}$ for $2 \mathrm{~h}$ in the dark. The absorbance values were measured at $405 \mathrm{~nm}$ with a spectrophotometer (Bio-Rad Laboratories, Inc.).
Western blot analysis. PC12 cells $\left(1 \times 10^{6}\right)$ were collected, washed and lysed with radioimmunoprecipitation assay buffer. The protein concentration was determined using a BCA Protein Assay kit (Thermo Fisher Scientific, Inc.). The protein samples $(30 \mu \mathrm{g})$ were separated by $10 \%$ SDS-PAGE and transferred to a polyvinylidene difluoride membrane (EMD Millipore, Billerica, MA, USA). After blocking with 5\% nonfat dry milk, the membrane was incubated overnight at $4^{\circ} \mathrm{C}$ with primary antibodies [1:1,000 dilution; rabbit antibodies to cleaved-caspase-3 (9664), caspase-3 (9665), phosphorylated (p)-Akt (Ser473) (4058), Akt (4685) and GAPDH (5174); all from Cell Signaling Technology, Inc., Beverly, MA, USA; rabbit antibodies to Bax (ab32503) and Bcl-2 (ab59348); both from Abcam, Cambridge, MA, USA]. The blotted membranes were then washed and probed with horseradish peroxidase-conjugated secondary antibodies (1:2,000 dilution; anti-rabbit) (7074; Cell Signaling Technology, Inc.). Immunoreactive bands were visualized using an enhanced chemiluminescence kit (32106; Thermo Fisher Scientific, Inc.). Images were captured using a scanner (Amersham Life Science, Arlington Heights, IL, USA). Intensities of protein bands were quantified using Image J software version 1.44 (National Institutes of Health, Bethesda, MD, USA).

Statistical analysis. Values are expressed as the mean \pm standard deviation. Differences between two groups were evaluated using Student's t-test. One-way analysis of variance was used for multi-group comparison. $\mathrm{P}<0.05$ was considered to indicate a statistically significant difference. Results were analyzed using SPSS 13.0 statistical software (SPSS, Inc., Chicago, IL, USA).

\section{Results}

Puerarin attenuates TNF- $\alpha$-induced cytotoxicity in PC12 cells. PC12 cells were treated with different concentrations of puerarin $(1-100 \mu \mathrm{M})$ for $24 \mathrm{~h}$. As shown in Fig. 1A, puerarin had no cytotoxicity on PC12 cells. Based on the inhibitory rate of TNF- $\alpha$ on PC12 cells $\left(\mathrm{IC}_{50}=2.856 \times 10^{5} \mathrm{U} / 1\right.$; Table I), $3 \times 10^{5} \mathrm{U} / 1 \mathrm{TNF}-\alpha$ was used to induce apoptosis in PC12 cells as an in vitro model of neuroinflammation-associated neuronal apoptosis. Of note, pre-treatment with puerarin $(25$ and $50 \mu \mathrm{M})$ significantly attenuated TNF- $\alpha$-induced cytotoxicity $(\mathrm{P}<0.05$; Fig. 1B).

Puerarin prevents TNF- $\alpha$-induced apoptosis in PC12 cells. As shown in Fig. 2, Annexin V/PI staining and flow cytometric analysis revealed that TNF- $\alpha$ obviously increased the apoptotic rate of $\mathrm{PC} 12$ cells $(25.25 \pm 1.46$ vs. $6.56 \pm 1.18 \%$ in the control group; $\mathrm{P}<0.001)$. Pre-treatment with puerarin at 25 or $50 \mu \mathrm{M}$ significantly prevented TNF- $\alpha$-induced apoptosis (apoptotic rate, $14.98 \pm 1.05$ or $14.26 \pm 1.12$ vs. $25.25 \pm 1.46 \%$ in TNF- $\alpha$ group; $\mathrm{P}<0.05$; Fig. 2).

Puerarin suppresses enzymatic activity of caspase-3 and -9. In line with the results on the apoptotic rate, the enzymatic activity of caspase-3, a marker of apoptosis, was found to be markedly increased in TNF- $\alpha$-treated cells. Pre-treatment with puerarin $(25$ and $50 \mu \mathrm{M})$ significantly suppressed TNF- $\alpha$-induced enzymatic activity of caspase-3 (Fig. 3A). Furthermore, the 
A

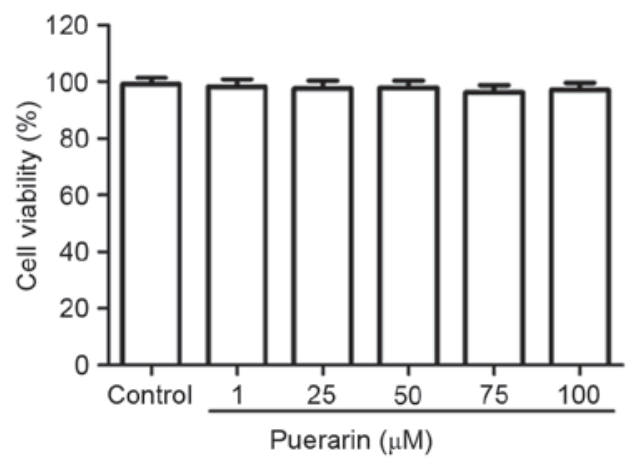

B

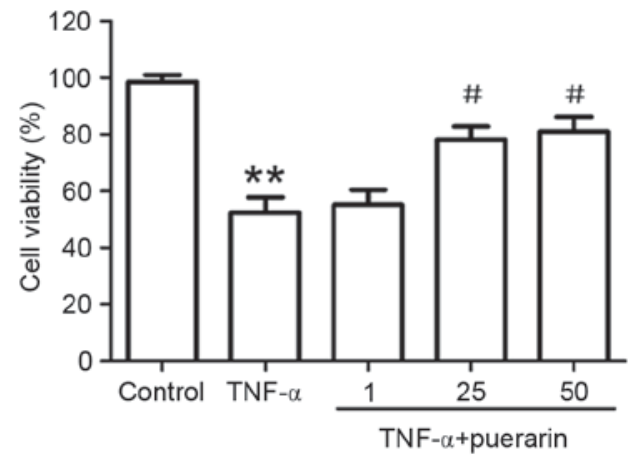

Figure 1. Effect of puerarin on TNF- $\alpha$-induced cytotoxicity in PC12 cells. (A) PC12 cells were treated with different concentrations of puerarin (1-100 $\mu \mathrm{M})$ for $24 \mathrm{~h}$. (B) PC12 cells were pre-treated with different concentrations of puerarin $(1,25$ and $50 \mu \mathrm{M})$ for $2 \mathrm{~h}$ prior to exposure to TNF- $\alpha$ (3x10 $\mathrm{U} / 1)$. Cell viability was determined by an MTT assay. Values are expressed as the mean \pm standard deviation $(\mathrm{n}=3)$. ${ }^{* * *} \mathrm{P}<0.01 \mathrm{vs}$. control; ${ }^{*} \mathrm{P}<0.05$ vs. TNF- $\alpha$. TNF, tumor necrosis factor.

enzymatic activity of caspase-9 in TNF- $\alpha$-treated PC12 cells was investigated. As shown in Fig. 3B, puerarin $(50 \mu \mathrm{M})$ distinctly restrained TNF- $\alpha$-induced activation of caspase- 9 , suggesting that the mitochondrial apoptotic pathway may be involved in the suppressive effect of puerarin.

Puerarin inhibits TNF- $\alpha$-induced increases in Bax/Bcl-2 ratio and caspase-3 activation. As shown in Fig. 4A, TNF- $\alpha$ significantly induced the expression of Bax and inhibited that of Bcl-2, which was consistent with the fact that TNF- $\alpha$ induced apoptosis in PC12 cells. The Bax/Bcl-2 ratio increased by $\sim 25$-fold upon treatment with TNF- $\alpha$, while in cells that had been pre-treated with 25 or $50 \mu \mathrm{M}$ puerarin, the Bax/Bcl-2 ratio was significantly decreased to $\sim 16$ - and 3 -fold of that in the control group, respectively $(\mathrm{P}<0.05)$. In addition, TNF- $\alpha$ evidently induced the activation of cleaved caspase-3 protein. Puerarin (25 and $50 \mu \mathrm{M})$ significantly decreased the levels of cleaved caspase- 3 , demonstrating its inhibitive function on TNF- $\alpha$-induced apoptosis $(\mathrm{P}<0.05$; Fig. 4B).

Puerarin inhibits TNF- $\alpha$-induced decreases of p-Akt (Ser473). In addition, the detailed mechanism underlying the suppressive effect of puerarin was investigated. TNF- $\alpha$ significantly suppressed the phosphorylation of Akt (Ser473) compared with that in the control group $(\mathrm{P}<0.001)$. Conversely, pre-treatment with puerarin prior to exposure to TNF- $\alpha$ significantly promoted the activation of Akt by its phosphorylation at Ser473 ( $\mathrm{P}<0.05$; Fig. 5A). Of note, the PI3K inhibitor LY294002, applied to the cells prior to puerarin treatment, obviously reversed the p-Akt activation achieved by puerarin treatment $(\mathrm{P}<0.05)$, suggesting that puerarin prevented TNF- $\alpha$-induced apoptosis in PC12 cells via activation of the PI3K/Akt signaling pathway (Fig. 5B).

\section{Discussion}

It is well-known that TNF- $\alpha$ has an important role in the neuroinflammatory response linked with several neurodegenerative diseases, including Parkinson's disease, Alzheimer's disease and multiple sclerosis, which are associated with neuronal apoptosis (14). The present study suggested that puerarin prevented TNF- $\alpha$-induced apoptosis in PC12 cells
Table I. Inhibitory rate of TNF- $\alpha$ on the proliferation of PC12 cells.

\begin{tabular}{lcc}
\hline TNF- $\alpha(\mathrm{U} / \mathrm{l})$ & OD570 & Inhibitory rate $(\%)$ \\
\hline 0 & $0.996 \pm 0.005$ & 0 \\
$1 \times 10^{4}$ & $0.968 \pm 0.016$ & $9.716 \pm 0.563$ \\
$5 \times 10^{4}$ & $0.782 \pm 0.023$ & $10.038 \pm 0.862$ \\
$1 \times 10^{5}$ & $0.604 \pm 0.018$ & $13.718 \pm 1.056$ \\
$5 \times 10^{5}$ & $0.427 \pm 0.026$ & $53.582 \pm 1.256$ \\
$1 \times 10^{6}$ & $0.295 \pm 0.012$ & $84.535 \pm 0.682$ \\
\hline
\end{tabular}

Values are expressed as the mean \pm standard deviation. OD570, optical density at $570 \mathrm{~nm}$; TNF, tumor necrosis factor.

through anti-apoptotic mechanisms, including activation of the PI3K/Akt signaling pathway, suggesting its promising prospect as a neuroprotective drug candidate.

Puerarin, the major bioactive constituent of Radix puerariae, is widely used in China for the treatment of cardiovascular diseases and diabetes (15). The neuroprotective potential of puerarin has been comprehensively evaluated in cell cultures and rodent models of several neurodegenerative diseases (16). A previous study showed that puerarin suppressed 6-hydroxydopamine-induced nitric oxide (NO) production and neurotoxicity in $\mathrm{PC} 12$ cells and primary rat midbrain neurons, which was attributed to the activation of arginase- 2 in the L-arginine-NO pathway (17). In addition, puerarin significantly prevented 1-methyl-4-phenylpyridinium $\left(\mathrm{MPP}^{+}\right)$-induced neurotoxicity in $\mathrm{PC} 12$ cells through inhibition of the c-Jun N-terminal kinase (JNK) signaling pathway (18). Furthermore, puerarin exerted a protective effect against epilepsy-induced brain injury through antioxidant and anti-apoptotic mechanisms (19). In line with these findings, puerarin also prevented amyloid $\beta$-induced neurotoxicity in PC12 cells. In the present study, various concentrations of puerarin $(1-100 \mu \mathrm{M})$ showed no cytotoxicity (Fig. 1A), while TNF- $\alpha\left(3 \times 10^{5} \mathrm{U} / \mathrm{l}\right)$ obviously inhibited the proliferation of PC12 cells (Fig. 1B). Of note, puerarin (25 and $50 \mu \mathrm{M})$ significantly attenuated TNF- $\alpha$-induced apoptosis of PC12 cells. To the best of our knowledge, the present study 

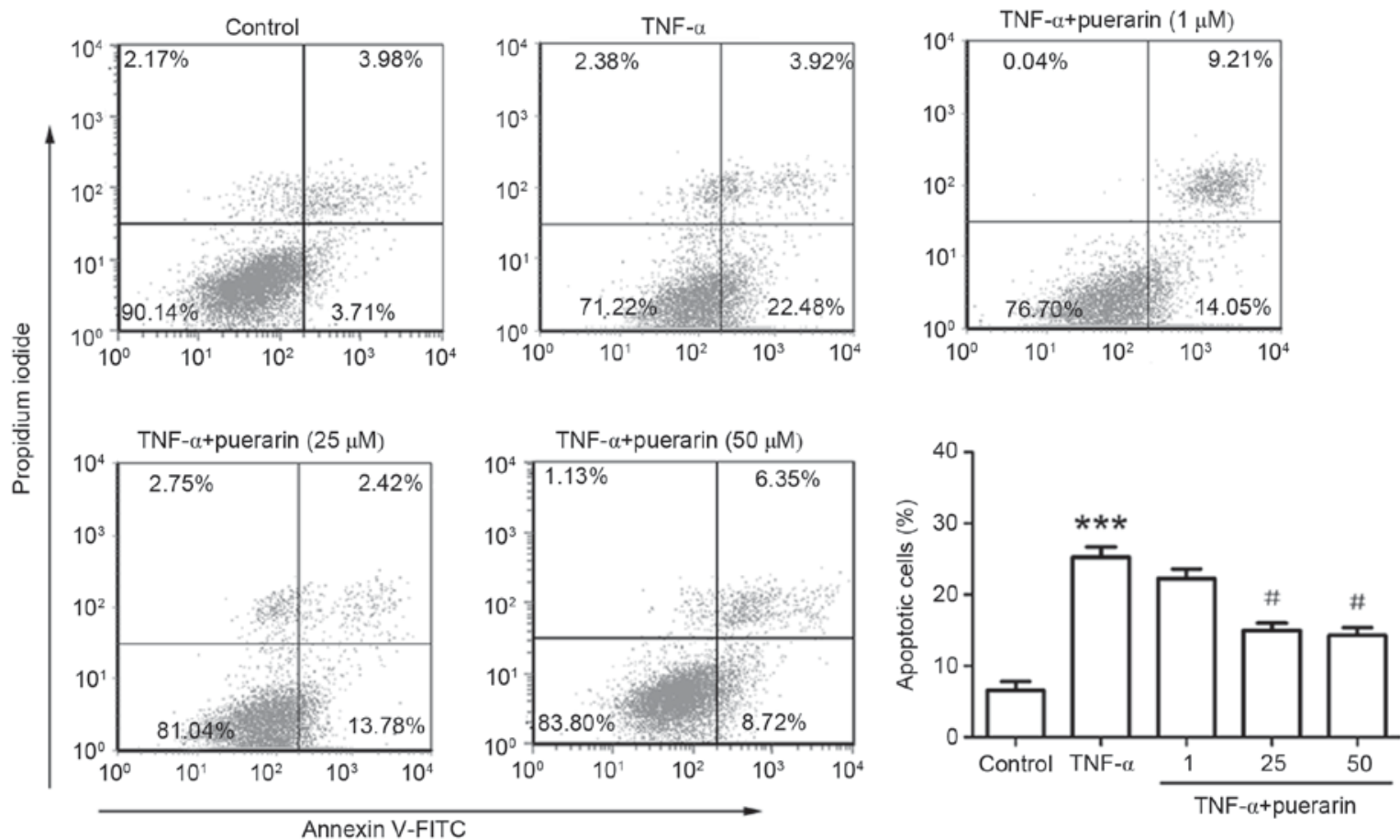

Figure 2. Effect of puerarin on TNF- $\alpha$-induced apoptosis of PC12 cells. PC12 cells were pretreated with different concentrations of puerarin $(1,25$ and $50 \mu \mathrm{M})$ for $2 \mathrm{~h}$ prior to exposure to TNF- $\alpha\left(3 \times 10^{5} \mathrm{U} / 1\right)$. Cell apoptosis was detected by flow cytometric analysis after staining with Annexin V and PI, and apoptotic cells (Annexin $\mathrm{V}^{+} \mathrm{PI}-$ and Annexin $\left.\mathrm{V}^{+} \mathrm{PI}^{+}\right)$were shown. Values are expressed as the mean \pm standard deviation $(\mathrm{n}=3){ }^{* * *} \mathrm{P}<0.001 \mathrm{vs}$. control; ${ }^{*} \mathrm{P}<0.05 \mathrm{vs}$. TNF- $\alpha$. TNF, tumor necrosis factor; PI, propidium iodide; FITC, fluorescein isothiocyanate.
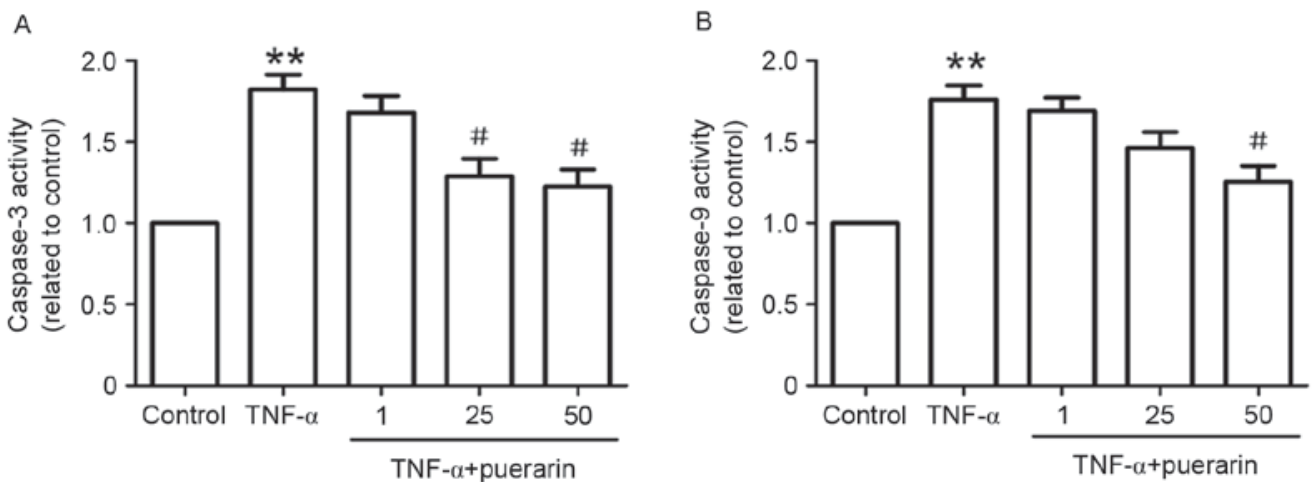

Figure 3. Effect of puerarin on enzymatic activity of caspase-3 and -9. PC12 cells were pre-treated with various concentrations of puerarin (1,25 and $50 \mu \mathrm{M})$ for $2 \mathrm{~h}$ prior to exposure to TNF- $\alpha\left(3 \times 10^{5} \mathrm{U} / \mathrm{l}\right)$. The activity of (A) caspase-3 and (B) caspase-9 activity was assessed via enzymatic assays. Values are expressed as the mean \pm standard deviation $(\mathrm{n}=3) .{ }^{* *} \mathrm{P}<0.01$ vs. control; ${ }^{*} \mathrm{P}<0.05$ vs. TNF- $\alpha$. TNF, tumor necrosis factor.

was the first to report that puerarin reduces $\mathrm{TNF}-\alpha$-induced neurotoxicity in PC12 cells.

Apoptosis is a programmed cell death and is induced via well-characterized intrinsic and extrinsic pathways. The intrinsic pathway is initiated in response to stress within the cell, such as DNA damage and endoplasmic reticulum stress. The extrinsic pathway is initiated through activation of pro-apoptotic receptors on the cell surface, which are activated by pro-apoptotic ligands (20). Accumulating evidence has indicated that puerarin exerts its neuroprotective effect by inhibition of neuronal apoptosis. In PC12 cells treated with amyloid $\beta$, suppression of apoptosis by puerarin was shown to involve modulation of the levels of pro- and anti-apoptotic proteins, including Bax, phosphorylated Bcl-2-associated death promoter and Bcl-2 (21). Puerarin significantly prevented
$\mathrm{MPP}^{+}$-induced cytostatic activities, caspase-3 activation and DNA fragmentation in PC12 cells via suppressing the activation of caspase- 9 and release of cytochrome $c$ (22). Consistent with these previous findings, puerarin inhibited TNF- $\alpha$-induced increases in the $\mathrm{Bax} / \mathrm{Bcl}-2$ ratio and caspase- 3 activation in the present study. The suppressive effect of puerarin was further demonstrated by the decreased enzymatic activity of caspase-3, which acts as an essential executor and biomarker of apoptosis in mammalian cells. In addition, the involvement of enzymatic activity of caspase- 9 suggested that TNF- $\alpha$-induced neuronal apoptosis and its inhibition by puerarin proceed via the mitochondrial apoptotic pathway.

Although the precise molecular mechanisms by which puerarin exerts its anti-apoptotic effects remain to be fully clarified, the results of the present and previous studies indicated 
A
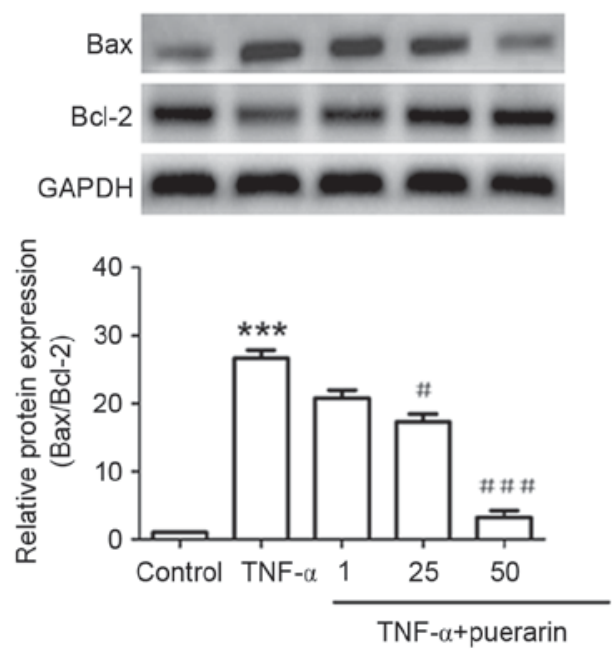

B

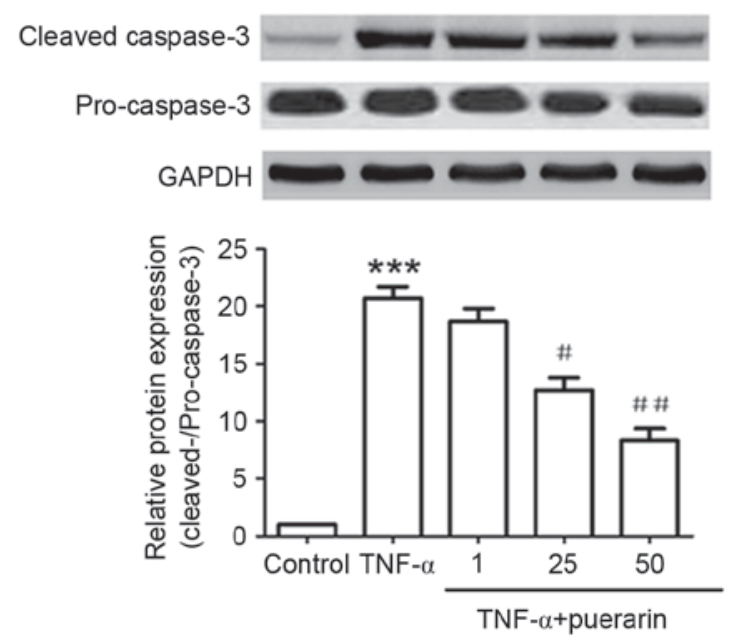

Figure 4. Effect of puerarin on the expression of Bax/Bcl-2 and caspase-3 protein. $\mathrm{PC} 12$ cells were pre-treated with various concentrations of puerarin $(1,25$ and $50 \mu \mathrm{M})$ for $2 \mathrm{~h}$ prior to exposure to TNF- $\alpha\left(3 \times 10^{5} \mathrm{U} / \mathrm{l}\right)$. (A) Protein expression of Bax and Bcl-2 was detected, and the Bax/Bcl-2 ratio was evaluated. (B) Protein levels of cleaved caspase-3 and pro-caspase-3 were detected by western blotting. Representative western blot images are shown. Values are expressed as the mean \pm standard deviation $(\mathrm{n}=3)$. ${ }^{* * *} \mathrm{P}<0.001$ vs. control; ${ }^{*} \mathrm{P}<0.05,{ }^{\# \#} \mathrm{P}<0.01$ and ${ }^{\# \# \# "} \mathrm{P}<0.001 \mathrm{vs}$. TNF- $\alpha$. TNF, tumor necrosis factor; Bcl-2, B-cell lymphoma 2; Bax, Bcl-2-associated X protein.

A
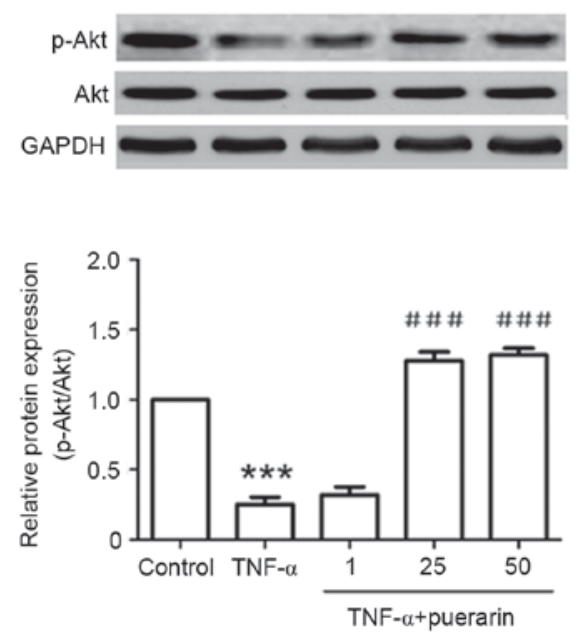

B
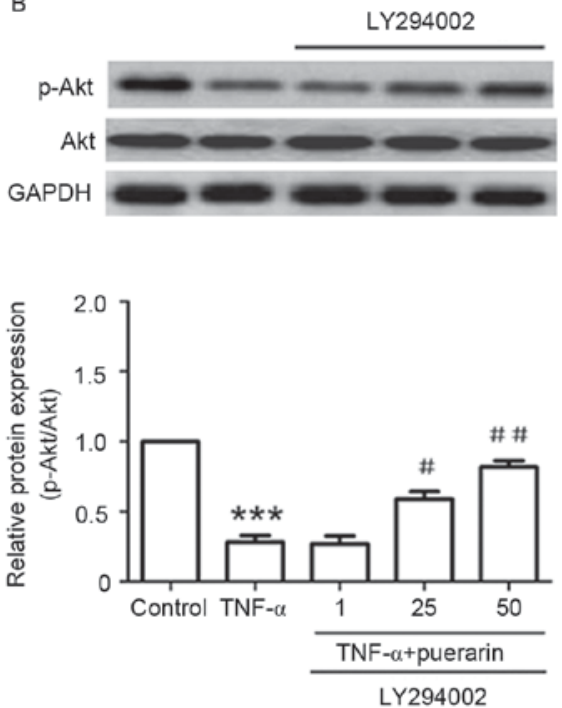

Figure 5. Puerarin inhibits TNF- $\alpha$-induced decreases in p-Akt (Ser473) in PC12 cells. PC12 cells were pre-treated with various concentrations of puerarin $(1,25$ and $50 \mu \mathrm{M})$ for $2 \mathrm{~h}$ prior to exposure to TNF- $\alpha\left(3 \times 10^{5} \mathrm{U} / 1\right)$. (A) Protein levels of Akt and p-Akt (Ser473) were assessed by western blotting. (B) LY294002 $(10 \mu \mathrm{M})$ was used prior to addition of puerarin for $2 \mathrm{~h}$. In the presence of the phosphoinositide-3 kinase inhibitor LY294002, protein levels of Akt and p-Akt (Ser473) were detected by western blotting. The $\mathrm{p}$-Akt/Akt ratio was determined. Values are expressed as the mean \pm standard deviation $(\mathrm{n}=3) .{ }^{* * * *} \mathrm{P}<0.001$ vs. control; ${ }^{\#} \mathrm{P}<0.05,{ }^{\# \#} \mathrm{P}<0.01$ and ${ }^{\# \# \#} \mathrm{P}<0.001$ vs. TNF- $\alpha$. TNF, tumor necrosis factor; p-Akt, phosphorylated Akt.

that the PI3K/Akt signaling pathway has a pivotal role in the process. A recent study indicated that puerarin restrained the progression of cardiac hypertrophy and apoptosis, which was probably mediated by the blockade of PI3K/Akt and JNK signaling pathways (23). In diabetic mice, puerarin exerted a protective effect on pancreatic $\beta$-cell function and survival, which was mediated via the PI3K/Akt pathway (24). Treatment with puerarin effectively inhibited lead-induced apoptosis in the kidney, which was associated with its antioxidant activity and its ability to modulate the PI3K/Akt/endothelial NO synthase signaling pathway (25). Furthermore, puerarin-mediated attenuation of amyloid $\beta$-induced microglial apoptosis was dependent upon activation of the PI3K survival pathway and phosphorylation of Akt (26). Puerarin inhibited lead acetate-induced oxidative stress in PC12 cells, associated with inhibiting PI3K and Akt phosphorylation, through increasing glutathione synthesis (27). In line with these results, the present study also showed that puerarin inhibited TNF-induced apoptosis and decreases of Akt activation in PC12 cells, which was associated with its neuroprotective effect.

In conclusion, the present study showed that puerarin prevented TNF- $\alpha$-induced apoptosis of PC12 cells via the PI3K/Akt signaling pathway, resulting in inhibition of TNF- $\alpha$-mediated caspase- 3 activation and increases in 
the $\mathrm{Bax} / \mathrm{Bcl}-2$ ratio. These findings partly explained the mechanisms underlying the neuroprotective effect of puerarin, suggesting its potential application in the clinical treatment of neuroinflammation involved in neurodegenerative diseases such as Parkinson's disease, Alzheimer's disease and multiple sclerosis.

\section{References}

1. Carson MJ, Thrash JC and Walter B: The cellular response in neuroinflammation: The role of leukocytes, microglia and astrocytes in neuronal death and survival. Clin Neurosci Res 6: 237-245, 2006

2. Chu WM: Tumor necrosis factor. Cancer Lett 328: 222-225, 2013.

3. Okouchi M, Ekshyyan O, Maracine M and Aw TY: Neuronal apoptosis in neurodegeneration. Antioxid Redox Signal 9: 1059-1096, 2007.

4. Martin TF and Grishanin RN: PC12 cells as a model for studies of regulated secretion in neuronal and endocrine cells. Methods Cell Biol 71: 267-286, 2003.

5. Wang X, Liu J, Jin NA, Xu D, Wang J, Han Y and Yin N: Fructus Corni extract-induced neuritogenesis in PC12 cells is associated with the suppression of stromal interaction molecule 1 expression and inhibition of $\mathrm{Ca}^{2+}$ influx. Exp Ther Med 9: 1773-1779, 2015.

6. Yin N, Hong X, Han Y, Duan Y, Zhang Y and Chen Z: Cortex Mori Radicis Extract induces neurite outgrowth in PC12 cells activating ERK signaling pathway via inhibiting $\mathrm{Ca}(2+)$ influx Int J Clin Exp Med 8: 5022-5032, 2015.

7. Maji AK, Pandit S, Banerji P and Banerjee D: Pueraria tuberosa: A review on its phytochemical and therapeutic potential. Nat Prod Res 28: 2111-2127, 2014.

8. Xie N, Wang C, Lian Y, Wu C, Zhang H and Zhang Q: Puerarin protects hippocampal neurons against cell death in pilocarpine-induced seizures through antioxidantand anti-apoptotic mechanisms. Cell Mol Neurobiol 34: 1175-1182, 2014.

9. Wang C, Xie N, Zhang H, Li Y and Wang Y: Puerarin protects against $\beta$-amyloid-induced microglia apoptosis via a PI3K-dependent signaling pathway. Neurochem Res 39: 2189-2196, 2014.

10. Liu LJ, Liu LQ, Bo T, Li SJ, Zhu Z, Cui RR and Mao DA: Puerarin suppress apoptosis of human osteoblasts via ERK signaling pathway. Int J Endocrinol 2013: 786574, 2013.

11. Yuan Y, Zong J, Zhou H, Bian ZY, Deng W, Dai J, Gan HW, Yang Z, Li H and Tang QZ: Puerarin attenuates pressure overload-induced cardiac hypertrophy. J Cardiol 63: 73-81, 2014.

12. Zhang HY, Liu YH, Wang HQ, Xu JH and Hu HT: Puerarin protects PC12 cells against beta-amyloid-induced cell injury. Cell Biol Int 32: 1230-1237, 2008.

13. Zhang Q, Huang WD, Lv XY and Yang YM: Puerarin protects differentiated $\mathrm{PC} 12$ cells from $\mathrm{H}_{2} \mathrm{O}_{2}$-induced apoptosis through the PI3K/Akt signalling pathway. Cell Biol Int 36: 419-426, 2012.
14. Frankola KA, Greig NH, Luo W and Tweedie D: Targeting TNF- $\alpha$ to elucidate and ameliorate neuroinflammation in neurodegenerative diseases. CNS Neurol Disord Drug Targets 10: 391-403, 2011.

15. Zhang Z, Lam TN and Zuo Z: Radix Puerariae: An overview of its chemistry, pharmacology, pharmacokinetics, and clinical use. J Clin Pharmacol 53: 787-811, 2013.

16. Zhou YX, Zhang $\mathrm{H}$ and Peng C: Puerarin: A review of pharmacological effects. Phytother Res 28: 961-975, 2014.

17. Zhao J, Cheng Y, Yang C, Lau S, Lao L, Shuai B, Cai J and Rong J: Botanical drug puerarin attenuates 6-hydroxydopamine (6-OHDA)-induced neurotoxicity via upregulating mitochondrial enzyme arginase-2. Mol Neurobiol 53: 2200-2201, 2016.

18. Bo J, Ming BY, Gang LZ, Lei C and Jia AL: Protection by puerarin against MPP+-induced neurotoxicity in PC12 cells mediated by inhibiting mitochondrial dysfunction and caspase-3-like activation. Neurosci Res 53: 183-188, 2005.

19. Xie N, Wang C, Lian Y, Wu C, Zhang H and Zhang Q: Puerarin protects hippocampal neurons against cell death in pilocarpine-induced seizures through antioxidant and anti-apoptotic mechanisms. Cell Mol Neurobiol 34: 1175-1182, 2014.

20. Lockshin RA and Zakeri Z: Programmed cell death and apoptosis: Origins of the theory. Nat Rev Mol Cell Biol 2: 545-550, 2001.

21. Xing G, Dong M, Li X, Zou Y, Fan L, Wang X, Cai D, Li C, Zhou L, Liu J and Niu Y: Neuroprotective effects of puerarin against beta-amyloid-induced neurotoxicity in PC12 cells via a PI3K-dependent signaling pathway. Brain Res Bull 85: 212-218, 2011.

22. Wang G, Zhou L, Zhang Y, Dong M, Li X, Liu J and Niu Y: Implication of the c-Jun-NH2-terminal kinase pathway in the neuroprotective effect of puerarin against 1-methyl-4-phenylpyridinium (MPP+)-induced apoptosis in PC-12 cells. Neurosci Lett 487: 88-93, 2011.

23. Hwang YP and Jeong HG: Mechanism of phytoestrogen puerarin-mediated cytoprotection following oxidative injury: Estrogen receptor-dependent up-regulation of PI3K/Akt and HO-1. Toxicol Appl Pharmacol 233: 371-381, 2008.

24. Li Z, Shangguan Z, Liu Y, Wang J, Li X, Yang S and Liu S: Puerarin protects pancreatic $\beta$-cell survival via PI3K/Akt signaling pathway. J Mol Endocrinol 53: 71-79, 2014.

25. Liu CM, Ma JQ and Sun YZ: Puerarin protects rat kidney from lead-induced apoptosis by modulating the PI3K/Akt/eNOS pathway. Toxicol Appl Pharmacol 258: 330-342, 2012.

26. Haque Bhuiyan MM, Mohibbullah M, Hannan MA, Hong YK, Han CH, Kim YK and Moon IS: The neuritogenic and synaptogenic effects of the ethanolic extract of radix Puerariae in cultured rat hippocampal neurons. J Ethnopharmacol 173: $172-182,2015$.

27. Li C, Pan Z, Xu T, Zhang C, Wu Q and Niu Y: Puerarin induces the upregulation of glutathione levels and nuclear translocation of Nrf2 through PI3K/Akt/GSK-3 $\beta$ signaling events in PC12 cells exposed to lead. Neurotoxicol Teratol 46: 1-9, 2014. 